Journal of Universal Mathematics

Vol.3 No.1 PP.1-10 (2020)

ISSN-2618-5660

\title{
ON INTEGRAL INEQUALITIES FOR INVEX FUNCTIONS SATISFYING LIPSCHITZIAN REQUIREMENT
}

\author{
SEDA KILINÇ, ABDULLAH AKKURT, AND HÜSEYIN YILDIRIM
}

\begin{abstract}
Some new type of integral inequalities for functions from the Lipschitz class are obtained. These results involve some different types of integral averages for Lipschitzian functions. Special cases which are naturally included in the main results of the paper are also discussed.
\end{abstract}

\section{INTRODUCTION}

This study is about getting for Lipschitz functions of some inequalities that are obtained for Harmonic convex functions. At first,some basic definitions and theorems related to convexity, invex set, Lipschitz functions and important inequalities are given. Then some results in literature for lipschitz functons of invex type inequalities obtained for convex and harmonic convex functions are given.

In research findings of study, we have new inequalities for invex functions satisfying Lipschitz functions by means of inequalities which are used for Harmonic convex functions.

Finally, some special means applications of these inequalities are given.

\section{Preliminaries}

A function $f: I=[a, b] \rightarrow \mathbb{R}$ is said to be convex function in the classical sense, if the following inequality holds:

$$
f(t x+(1-t) y) \leq t f(x)+(1-t) f(y)
$$

$\forall x, y \in I, t \in[0,1]$.

A function $f$ such that

$$
|f(x)-f(y)| \leq M|x-y|
$$

for all $x$ and $y$, where $M$ is a constant independent of $x$ and $y$, is called a Lipschitz function. For example, any function with a bounded first derivative must be Lipschitz.

The Hermite-Hadamard inequality provides us an estimate of the mean value of a continuous convex function. This inequality also proves us a necessary and sufficient

2000 Mathematics Subject Classification. 26A33; 26D10; 26D15; 41A55.

Key words and phrases. Fractional Integral, Riemann-Liouville Fractional Integral, Integral Inequalities. 
condition for a function to be convex. The classic version of this inequality reads as:

Let $f: I \rightarrow \mathbb{R}$ be a convex function, then,

$$
f\left(\frac{a+b}{2}\right) \leq \frac{1}{b-a} \int_{a}^{b} f(x) \leq \frac{f(a)+f(b)}{2}
$$

$f: I \rightarrow \mathbb{R}$ is said to be harmonically convex, if

$$
f\left(\frac{x y}{t x+(1-t) y}\right) \leq t f(y)+(1-t) f(x)
$$

for all $x, y \in I$ and $t \in[0,1]$. If this inequality is reversed, then the function $f$ is said to be harmonically concave.

Definition 2.1. Let $I \subset \mathbb{R}$ be a harmonically convex function and $a, b \in I$ with $a<b$, if $f \in L[a, b]$ then the following inequalities hold:

$$
f\left(\frac{2 a b}{a+b}\right) \leq \frac{a b}{b-a} \int_{a}^{b} \frac{f(x)}{x^{2}} d x \leq \frac{f(a)+f(b)}{2} .
$$

A set $K$ is said to be invex set with respect to $\eta(.,$.$) if$

$$
u+\tau \eta(u, v) \in K, \forall u, v \in K, \tau \in[0,1] .
$$

The invex set $K$ is also called $\eta$-connected set.

Definition 2.2. [16] A function $f$ is said to be invex with respect to arbitrary bifunction $\eta(.,$.$) if$

$$
f(u+\tau \eta(u, v)) \leq(1-t) f(u)+t f(v)
$$

The function $f$ is said to be preincave if and only if $f$ is invex. For $\eta(u, v)=u-v$ , the invex functions becomes convex functions in the classical sense.

Definition 2.3. [12] The beta function denoted by $\beta(m, n)$ is defined as;

$$
\beta(m, n)=\int_{0}^{1} x^{m-1}(1-x)^{n-1} d x .
$$

[12] The hypergeometric function denoted by ${ }_{2} F_{1}(a, b ; c ; z)$ is defined by the integral equality

$$
{ }_{2} F_{1}(a, b ; c ; z)=\frac{1}{\beta(b, c-b)} \int_{0}^{1} t^{b-1}(1-t)^{c-b-1}(1-z t)^{-a} d t
$$

for $c>b>0,|z|<1$.

Corollary 2.4. [11]If we take $x=a+t \eta(b, a), \eta(x, y) \geq x-y$ and $y=\frac{a b}{b-t \eta(b, a)}$ in (2.2), then following inequality holds for arbitrary $t \in[0,1]$, also we name as $M$-Lipschitzian ;

$$
\begin{aligned}
& \left|f(a+t \eta(b, a))-f\left(\frac{a b}{b-t \eta(b, a)}\right)\right| \\
& \leq M\left|a+t \eta(b, a)-\frac{a b}{b-t \eta(b, a)}\right| \\
& =M\left|\frac{(a+t \eta(b, a))(b-t \eta(b, a))-a b}{b-t \eta(b, a)}\right| \\
& =M\left|\frac{-a t \eta(b, a)+b t \eta(b, a)-t^{2} \eta^{2}(b, a)}{b-t \eta(b, a)}\right| \\
& =M\left|\frac{t(b-a) \eta(b, a)-t^{2} \eta^{2}(b, a)}{b-t \eta(b, a)}\right| \\
& \leq M \frac{t(1-t) \eta^{2}(b, a)}{b-t \eta(b, a)} .
\end{aligned}
$$




\section{Main Results}

In this section, we obtain some new inequalities for $M$-Lipschitzian functions.

Theorem 3.1. Let $f: I \subset(0, \infty) \rightarrow \mathbb{R}$ be an $M$-Lipschitzian mapping on interval $I$ and $a, b \in I$ with $a<b$. Then $\eta(.,):. K \times K \rightarrow \mathbb{R}^{n}$ following inequality holds;

$$
\begin{aligned}
& \left|\frac{1}{\eta(b, a)} \int_{a}^{a+\eta(b, a)} f(u) d u-\frac{a b}{\eta(b, a)} \int_{a}^{\frac{a b}{b-\eta(b, a)}} \frac{f(u)}{u^{2}} d u\right| \\
\leq & \frac{M \eta^{2}(b, a)}{6 b}{ }_{2} F_{1}\left(1,2 ; 4 ; \frac{\eta(b, a)}{b}\right) .
\end{aligned}
$$

Proof. Since $f$ is $M$-Lipschitzian function on interval $I, \forall x, y \in[a, b]$

$$
|f(x)-f(y)| \leq M|x-y| .
$$

From Corollary ??, we write

$$
\left|f(a+t \eta(b, a))-f\left(\frac{a b}{b-t \eta(b, a)}\right)\right| \leq M \frac{t(1-t) \eta^{2}(b, a)}{b-t \eta(b, a)}
$$

If we take integral the last inequality on $t \in[0,1]$, and use property of modulus, we get

$$
\begin{gathered}
\left|\int_{0}^{1} f(a+t \eta(b, a)) d t-\int_{0}^{1} f\left(\frac{a b}{b-t \eta(b, a)}\right) d t\right| \\
\leq \int_{0}^{1}\left|f(a+t \eta(b, a))-f\left(\frac{a b}{b-t \eta(b, a)}\right)\right| d t \\
\leq M \eta^{2}(b, a) \int_{0}^{1} \frac{t(1-t)}{b-t \eta(b, a)} d t \\
=M \eta^{2}(b, a) \int_{0}^{1} \frac{t(1-t)}{b\left[1-t \frac{\eta(b, a)}{b}\right]} d t \\
=\frac{M \eta^{2}(b, a)}{b}{ }_{2} F_{1}\left(1,2 ; 4 ; \frac{\eta(b, a)}{b}\right) \beta(2,2)
\end{gathered}
$$

If we take $u=a+t \eta(b, a)$ and $u=\frac{a b}{b-t \eta(b, a)}$ in the integrals on the left side of the last inequality respectively, we have the following inequalities;

$$
\begin{aligned}
& \left|\frac{1}{\eta(b, a)} \int_{a}^{a+\eta(b, a)} f(u) d u-\frac{a b}{\eta(b, a)} \int_{a}^{\frac{a b}{b-\eta(b, a)}} \frac{f(u)}{u^{2}} d u\right| \\
& \leq \frac{M \eta^{2}(b, a)}{6 b}{ }_{2} F_{1}\left(1,2 ; 4 ; \frac{\eta(b, a)}{b}\right)
\end{aligned}
$$

this completes the proof of theorem.

Remark 3.2 (11). If we take $\eta(b, a)=b-a$ from the above Theorem 1 , we obtain then following inequality holds;

$$
\begin{aligned}
& \left|\frac{1}{b-a} \int_{a}^{b} f(u) d u-\frac{a b}{b-a} \int_{a}^{b} \frac{f(u)}{u^{2}} d u\right| \\
\leq & \frac{M(b-a)^{2}}{6 b}{ }_{2} F_{1}\left(1,2 ; 4 ; \frac{b-a}{b}\right) .
\end{aligned}
$$

Definition 3.3. The $p$ logarithmic mean

$$
L_{p}(a, b)=\left\{\begin{array}{cc}
a, & \text { if } a=b \\
\frac{b^{p+1}-a^{p+1}}{(b-a)(p+1)} & \text { if } a \neq b
\end{array}\right.
$$

where $a, b>0$. 
Definition 3.4. The $\eta-p$ logarithmic mean

$$
L_{p, \eta}^{p}(a, b)=\left\{\begin{array}{cl}
a, & \text { if } a=b \\
\frac{[a+\eta(b, a)]^{p+1}-a^{p+1}}{\eta(b, a)(p+1)} & \text { if } a \neq b
\end{array}\right.
$$

where $a, b>0$.If we take $\eta(b, a)=b-a$, we obtain clasical $p$ logarithmic mean.

Definition 3.5. The Geometric mean

$$
G=G(a, b)=\sqrt{a . b}
$$

Definition 3.6. The logarithmic mean

$$
L_{1}(a, b)\left\{\begin{array}{cc}
a, & \text { if } a=b \\
\frac{b-a}{\ln b-\ln a} & \text { if } a \neq b
\end{array}\right.
$$

where $a, b>0$.

Proposition 1. Let $p \in(1, \infty) \backslash\{2\}$ and $a, b \in \mathbb{R}$ with $o<a<b$. Then $\eta(.,$.$) :$ $K \times K \rightarrow \mathbb{R}^{n}$ following inequality holds;

$$
\begin{aligned}
& \left|L_{p, \eta}^{p}(a, b)-G^{2} L_{p-2, \eta}^{p-2}(a, b)\right| \\
& \leq \frac{M \eta^{2}(b, a)}{6 b}{ }_{2} F_{1}\left(1,2 ; 4 ; \frac{\eta(b, a)}{b}\right) .
\end{aligned}
$$

Proof. If the $f(x)=x^{p}$ convex mapping defined on interval $[a, b]$ is applied to the left side of the inequality in Theorem 1 , the inequality

$$
\begin{aligned}
& \left|\frac{1}{\eta(b, a)} \int_{a}^{a+\eta(b, a)} f(u) d u-\frac{a b}{\eta(b, a)} \int_{a}^{\frac{a b}{b-\eta(b, a)}} \frac{f(u)}{u^{2}} d u\right| \\
& =\left|\frac{1}{\eta(b, a)} \int_{a}^{a+\eta(b, a)} u^{p} d u-\frac{a b}{\eta(b, a)} \int_{a}^{\frac{a b}{b-\eta(b, a)}} \frac{u^{p}}{u^{2}} d u\right| \\
& \leq \frac{M \eta^{2}(b, a)}{6 b}{ }_{2} F_{1}\left(1,2 ; 4 ; \frac{\eta(b, a)}{b}\right)
\end{aligned}
$$

is obtained. If the integral is calculated,

$$
\begin{aligned}
& \left|\frac{1}{\eta(b, a)} \int_{a}^{a+\eta(b, a)} u^{p} d u-\frac{a b}{\eta(b, a)} \int_{a}^{\frac{a b}{b-\eta(b, a)}} \frac{u^{p}}{u^{2}} d u\right| \\
& =\left|\frac{[a+\eta(b, a)]^{p+1}-a^{p+1}}{\eta(b, a)(p+1)}-\frac{a b}{\eta(b, a)(p-1)}\left[\left(\frac{a b}{b-\eta(b, a)}\right)^{p-1}-a^{p-1}\right]\right| \\
& \leq \frac{M \eta^{2}(b, a)}{6 b}{ }_{2} F_{1}\left(1,2 ; 4 ; \frac{\eta(b, a)}{b}\right)
\end{aligned}
$$

where

$$
\begin{aligned}
& \left|\frac{1}{\eta(b, a)} \int_{a}^{a+\eta(b, a)} u^{p} d u-\frac{a b}{\eta(b, a)} \int_{a}^{\frac{a b}{b-\eta(b, a)}} \frac{u^{p}}{u^{2}} d u\right| \\
& =\left|L_{p, \eta}^{p}(a, b)-G^{2} L_{p-2, \eta}^{p-2}(a, b)\right| \\
& \leq \frac{M \eta^{2}(b, a)}{6 b}{ }_{2} F_{1}\left(1,2 ; 4 ; \frac{\eta(b, a)}{b}\right) .
\end{aligned}
$$

Proposition 2. Let $p \in(1, \infty) \backslash\{2\}$ and $a, b \in \mathbb{R}$ with $o<a<b$. Then $\eta(.,$.$) :$ $K \times K \rightarrow \mathbb{R}^{n}$ following inequality holds;

$$
\begin{aligned}
& \left|L_{\eta}^{-1}-\frac{a^{-2}}{[b-\eta(b, a)]^{-2}} G^{-2} A_{\eta}\right| \\
& \leq \frac{M \eta^{2}(b, a)}{6 b}{ }_{2} F_{1}\left(1,2 ; 4 ; \frac{\eta(b, a)}{b}\right) .
\end{aligned}
$$


Proof. If the $f(x)=\frac{1}{x}$ convex mapping defined on interval $[a, b]$ is applied to the left side of the inequality in Theorem 1, the inequality

$$
\begin{aligned}
& \left|\frac{1}{\eta(b, a)} \int_{a}^{a+\eta(b, a)} f(u) d u-\frac{a b}{\eta(b, a)} \int_{a}^{\frac{a b}{b-\eta(b, a)}} \frac{f(u)}{u^{2}} d u\right| \\
& =\left|\frac{1}{\eta(b, a)} \int_{a}^{a+\eta(b, a)} u^{-1} d u-\frac{a b}{\eta(b, a)} \int_{a}^{\frac{a b}{b-\eta(b, a)}} \frac{u^{-1}}{u^{2}} d u\right| \\
& \leq \frac{M \eta^{2}(b, a)}{6 b}{ }_{2} F_{1}\left(1,2 ; 4 ; \frac{\eta(b, a)}{b}\right)
\end{aligned}
$$

is obtained. If the integral is calculated,

$$
\begin{gathered}
\left|\frac{1}{\eta(b, a)} \ln \right| \frac{a+\eta(b, a)}{a}\left|+\frac{a b}{2 \eta(b, a)}\left[\left(\frac{a b}{b-\eta(b, a)}\right)^{-2}-a^{-2}\right]\right| \\
\leq \frac{M \eta^{2}(b, a)}{6 b}{ }_{2} F_{1}\left(1,2 ; 4 ; \frac{\eta(b, a)}{b}\right) .
\end{gathered}
$$

where

$$
\begin{aligned}
& \left|L_{\eta}^{-1}-\frac{a^{-2}}{[b-\eta(b, a)]^{-2}} G^{-2} A_{\eta}\right| \\
& \leq \frac{M \eta^{2}(b, a)}{6 b}{ }_{2} F_{1}\left(1,2 ; 4 ; \frac{\eta(b, a)}{b}\right) .
\end{aligned}
$$

Theorem 3.7. Let $f: I \subset \mathbb{R} \rightarrow \mathbb{R}$ be an M-Lipschitzian function on interval $I$ and $a, b, x, y \in I$ with $a \leq x<y$ and $a<b$.Then $\eta(.,):. K \times K \rightarrow \mathbb{R}^{n}$ following inequality holds;

$$
\begin{aligned}
& \left|\frac{1}{\eta(b, a)} \int_{a}^{a+\eta(b, a)} f(u) d u-\frac{1}{\eta(y, x)} \int_{x}^{x+\eta(y, x)} f(u) d u\right| \\
& \leq M\left\{\frac{|\eta(b, a)-\eta(y, x)|}{2}+x-a\right\}
\end{aligned}
$$

Proof. Since $f$ is M-Lipschitzian function on interval $I, \forall x, w \in[a, b]$

$$
|f(x)-f(w)| \leq M|x-w| .
$$

Here, for arbitrary $t \in[0,1]$, if we take

$$
x=a+t \eta(b, a) \text { and } w=x+t \eta(y, x)
$$

then

$$
\begin{gathered}
|f(a+t \eta(b, a))-f(x+t \eta(y, x))| \\
\leq M|a+t \eta(b, a)-x-t \eta(y, x)| \\
=M|t(\eta(b, a)-\eta(y, x))+a-x| \\
\quad M\{t|\eta(b, a)-\eta(y, x)|+x-a\}
\end{gathered}
$$

If we take integral the last inequality on $t \in[0,1]$, and use property of modulus, we have

$$
\begin{aligned}
& \left|\int_{0}^{1} f(a+t \eta(b, a)) d t-\int_{0}^{1} f(x+t \eta(y, x)) d t\right| \\
& \leq \int_{0}^{1}|f(a+t \eta(b, a))-(x+t \eta(y, x))| d t \\
& \leq M|\eta(b, a)-\eta(y, x)| \int_{0}^{1} t d t+M|a-x| \int_{0}^{1} d t \\
& =M\left\{\frac{|\eta(b, a)-\eta(y, x)|}{2}+x-a\right\}
\end{aligned}
$$

If we make the change of variable $u=a+t \eta(b, a)$ and $u=x+t \eta(y, x)$ in the integrals on the left side of the last inequality respectively, we have the following inequalities;

$$
\begin{aligned}
& \left|\frac{1}{\eta(b, a)} \int_{a}^{a+\eta(b, a)} f(u) d u-\frac{1}{\eta(y, x)} \int_{x}^{x+\eta(y, x)} f(u) d u\right| \\
& \leq M\left\{\frac{|\eta(b, a)-\eta(y, x)|}{2}+x-a\right\}
\end{aligned}
$$


this completes the proof of the theorem.

Remark 3.8 (11). If we take $\eta(b, a)=b-a$ from the above theorem 2 , we obtain then following inequality holds;

$$
\begin{aligned}
& \left|\frac{1}{b-a} \int_{a}^{b} f(u) d u-\frac{1}{y-x} \int_{x}^{y} f(u) d u\right| \\
\leq & M\left\{\frac{|(b-a)-(y-x)|}{2}+x-a\right\}
\end{aligned}
$$

Proposition 3. Let $p>1$ and $a, b, x, y \in \mathbb{R}$ with $0<a \leq x<y$ and $a<b$. Then $\eta(.,):. K \times K \rightarrow \mathbb{R}^{n}$ following inequality holds;

$$
\begin{aligned}
& \left|L_{p, \eta}^{p}(a, b)-L_{p, \eta}^{p}(x, y)\right| \\
\leq & M\left\{\frac{|\eta(b, a)-\eta(y, x)|}{2}+x-a\right\}
\end{aligned}
$$

Proof. If the $f(x)=x^{p}$ convex mapping defined on interval $[a, b]$ is applied to the left side of the inequality in Theorem 2, the inequality

$$
\begin{aligned}
& \left|\frac{1}{\eta(b, a)} \int_{a}^{a+\eta(b, a)} f(u) d u-\frac{1}{\eta(y, x)} \int_{x}^{x+\eta(y, x)} f(u) d u\right| \\
& =\left|\frac{1}{\eta(b, a)} \int_{a}^{a+\eta(b, a)} u^{p} d u-\frac{1}{\eta(y, x)} \int_{x}^{x+\eta(y, x)} u^{p} d u\right| \\
& =\left|\frac{(a+\eta(b, a))^{p+1}-a^{p+1}}{\eta(b, a)(p+1)}-\frac{(x+\eta(y, x))^{p+1}-x^{p+1}}{\eta(y, x)(p+1)}\right| \\
& \leq M\left\{\frac{|\eta(b, a)-\eta(y, x)|}{2}+x-a\right\}
\end{aligned}
$$

where

$$
\begin{aligned}
& \left|L_{p, \eta}^{p}(a, b)-L_{p, \eta}^{p}(x, y)\right| \\
& \leq M\left\{\frac{|\eta(b, a)-\eta(y, x)|}{2}+x-a\right\}
\end{aligned}
$$

Proposition 4. Let $a, b, x, y \in \mathbb{R}$ with $0<a \leq x<y$ and $a<b$. Then $\eta(.,$.$) :$ $K \times K \rightarrow \mathbb{R}^{n}$ following inequality holds;

$$
\begin{aligned}
& \left|L_{\eta}^{-1}(a, b)-L_{\eta}^{-1}(x, y)\right| \\
& \leq M\left\{\frac{|\eta(b, a)-\eta(y, x)|}{2}+x-a\right\} .
\end{aligned}
$$

Proof. If the $f(x)=\frac{1}{x}$ convex mapping defined on interval $[a, b]$ is applied to the left side of the inequality in Theorem 2 , the inequality

$$
\begin{aligned}
& \left|\frac{1}{\eta(b, a)} \int_{a}^{a+\eta(b, a)} f(u) d u-\frac{1}{\eta(y, x)} \int_{x}^{x+\eta(y, x)} f(u) d u\right| \\
& =\left|\frac{1}{\eta(b, a)} \int_{a}^{a+\eta(b, a)} \frac{1}{u} d u-\frac{1}{\eta(y, x)} \int_{x}^{x+\eta(y, x)} \frac{1}{u} d u\right| \\
& =\left|\frac{1}{\eta(b, a)} \ln \right| \frac{a+\eta(b, a)}{a}\left|-\frac{1}{\eta(y, x)} \ln \right| \frac{x+\eta(y, x)}{x}|| \\
& =\left|L_{\eta}^{-1}(a, b)-L_{\eta}^{-1}(x, y)\right| \\
& \leq M\left\{\frac{|\eta(b, a)-\eta(y, x)|}{2}+x-a\right\} .
\end{aligned}
$$


Proposition 5. Let $a, b, x, y \in \mathbb{R}$ with $0<a \leq x<y$ and $a<b$. Then $\eta(.,$.$) :$ $K \times K \rightarrow \mathbb{R}^{n}$ following inequality holds;

$$
\begin{aligned}
& \left|\frac{e^{a+\eta(b, a)}-e^{a}}{\eta(b, a)}-\frac{e^{x+\eta(y, x)}-e^{x}}{\eta(y, x)}\right| \\
& \leq M\left\{\frac{|\eta(b, a)-\eta(y, x)|}{2}+x-a\right\} .
\end{aligned}
$$

Proof. If the $f(x)=e^{x}$ convex mapping defined on interval $[a, b]$ is applied to the left side of the inequality in Theorem 2, the inequality

$$
\begin{aligned}
& \left|\frac{1}{\eta(b, a)} \int_{a}^{a+\eta(b, a)} f(u) d u-\frac{1}{\eta(y, x)} \int_{x}^{x+\eta(y, x)} f(u) d u\right| \\
& =\left|\frac{1}{\eta(b, a)} \int_{a}^{a+\eta(b, a)} e^{u} d u-\frac{1}{\eta(y, x)} \int_{x}^{x+\eta(y, x)} e^{u} d u\right| \\
& =\left|\frac{e^{a+\eta(b, a)}-e^{a}}{\eta(b, a)}-\frac{e^{x+\eta(y, x)}-e^{x}}{\eta(y, x)}\right| \\
& \leq M\left\{\frac{|\eta(b, a)-\eta(y, x)|}{2}+x-a\right\} .
\end{aligned}
$$

Theorem 3.9. Let $f: I \subset \mathbb{R} \rightarrow \mathbb{R}$ be an M-Lipschitzian function on interval $I$ and $a, b, x, y \in I$ with $a \leq x<y$ and $a<b$.Then $\eta(.,):. K \times K \rightarrow \mathbb{R}^{n}$ following inequality holds;

$$
\begin{aligned}
& \left|\frac{a b}{\eta(b, a)} \int_{a}^{\frac{a b}{b+\eta(a, b)}} \frac{f(u)}{u^{2}} d u-\frac{x y}{\eta(y, x)} \int_{x}^{\frac{x y}{y+\eta(x, y)}} \frac{f(u)}{u^{2}} d u\right| \\
& \leq \frac{M}{\eta(a, b) \eta(x, y)}\left\{b y|a-x|\left[\frac{\eta(a, b) \eta(x, y)}{b \eta(x, y)-y \eta(a, b)}\left(\ln \left|\frac{\eta(x, y)+y}{y}\right|-\ln \left|\frac{\eta(a, b)+b}{b}\right|\right)\right]\right. \\
& \left.+\frac{|a b \eta(x, y)-x y \eta(a, b)|}{y \eta(a, b)-b \eta(x, y)}\left[y \eta(a, b) \ln \left|\frac{\eta(x, y)+y}{y}\right|-b \eta(x, y) \ln \left|\frac{\eta(a, b)+b}{b}\right|\right]\right\} .
\end{aligned}
$$

Proof. Since $f$ is M-Lipschitzian function on interval $I, \forall v, w \in[a, b]$

$$
|f(v)-f(w)| \leq M|v-w|
$$

Here, for arbitrary $t \in[0,1]$, if we take

$$
x=\frac{a b}{b+t \eta(a, b)} \text { and } w=\frac{x y}{y+t \eta(x, y)}
$$

then

$$
\begin{aligned}
& \left|f\left(\frac{a b}{b+t \eta(a, b)}\right)-f\left(\frac{x y}{y+t \eta(x, y)}\right)\right| \\
& \leq M\left|\frac{a b}{b+t \eta(a, b)}-\frac{x y}{y+t \eta(x, y)}\right| \\
& =M\left|\frac{a b(y+t \eta(x, y))-x y(b+t \eta(a, b))}{[b+t \eta(a, b)][y+t \eta(x, y)]}\right| \\
& =M\left|\frac{b y(a-x)+t(a b \eta(x, y)-x y \eta(a, b))}{[b+t \eta(a, b)](y+t \eta(x, y)]}\right| \\
& =M \frac{b y|a-x|+t|a b \eta(x, y)-x y \eta(a, b)|}{[b+t \eta(a, b)][y+t \eta(x, y)]} \\
& =\frac{M}{\eta(a, b) \eta(x, y)} \frac{b y|a-x+t|+|a b \eta(x, y)-x y \eta(a, b)|}{\left[t+\frac{b}{\eta(a, b)}\right]\left[t+\frac{y}{\eta(x, y)}\right]}
\end{aligned}
$$


If we take integral the last inequality on $t \in[0,1]$, and use property of modulus, we have

$$
\begin{aligned}
& \left|\int_{0}^{1} f\left(\frac{a b}{b+t \eta(a, b)}\right) d t-\int_{0}^{1} f\left(\frac{x y}{y+t \eta(x, y)}\right) d t\right| \\
& \leq \int_{0}^{1}\left|f\left(\frac{a b}{b+t \eta(a, b)}\right)-f\left(\frac{x y}{y+t \eta(x, y)}\right)\right| d t \\
& \leq \frac{M}{\eta(a, b) \eta(x, y)} \int_{0}^{1} \frac{b y|a-x|+t|a b \eta(x, y)-x y \eta(a, b)|}{\left[t+\frac{b}{\eta(a, b)}\right]\left[t+\frac{y}{\eta(x, y)}\right]} d t \\
& =\frac{M}{\eta(a, b) \eta(x, y)}\left\{b y|a-x| \int_{0}^{1} \frac{d t}{\left.\left[t+\frac{b}{\eta(a, b)}\right] \mid t+\frac{y}{\eta(x, y)}\right]}\right. \\
& \left.+|a b \eta(x, y)-x y \eta(a, b)| \int_{0}^{1} \frac{t d t}{\left.\left[t+\frac{b}{\eta(a, b)}\right] \mid t+\frac{y}{\eta(x, y)}\right]}\right\} \\
& =\frac{M}{\eta(a, b) \eta(x, y)}\left\{b y|a-x|\left[\frac{\eta(a, b) \eta(x, y)}{b \eta(x, y)-y \eta(a, b)}\left(\ln \left|\frac{\eta(x, y)+y}{y}\right|-\ln \left|\frac{\eta(a, b)+b}{b}\right|\right)\right]\right. \\
& \left.+\frac{|a b \eta(x, y)-x y \eta(a, b)|}{y \eta(a, b)-b \eta(x, y)}\left[y \eta(a, b) \ln \left|\frac{\eta(x, y)+y}{y}\right|-b \eta(x, y) \ln \left|\frac{\eta(a, b)+b}{b}\right|\right]\right\}
\end{aligned}
$$

If we make the change of variable $u=\frac{a b}{b+t \eta(a, b)}$ and $u=\frac{x y}{y+t \eta(x, y)}$ in the integrals on the left side of the last inequality respectively, we have the following inequalities;

$$
\begin{aligned}
& \left|\frac{a b}{\eta(b, a)} \int_{a}^{\frac{a b}{b+\eta(a, b)}} \frac{f(u)}{u^{2}} d u-\frac{x y}{\eta(y, x)} \int_{x}^{\frac{x y}{y+\eta(x, y)}} \frac{f(u)}{u^{2}} d u\right| \\
& \leq \frac{M}{\eta(a, b) \eta(x, y)}\left\{b y|a-x|\left[\frac{\eta(a, b) \eta(x, y)}{b \eta(x, y)-y \eta(a, b)}\left(\ln \left|\frac{\eta(x, y)+y}{y}\right|-\ln \left|\frac{\eta(a, b)+b}{b}\right|\right)\right]\right. \\
& \left.+\frac{|a b \eta(x, y)-x y \eta(a, b)|}{y \eta(a, b)-b \eta(x, y)}\left[y \eta(a, b) \ln \left|\frac{\eta(x, y)+y}{y}\right|-b \eta(x, y) \ln \left|\frac{\eta(a, b)+b}{b}\right|\right]\right\} .
\end{aligned}
$$

this completes the proof of the theorem.

Remark 3.10 (11). If we take $\eta(b, a)=b-a$ from the above Theorem 3 , we obtain then following inequality holds;

$$
\begin{aligned}
& \left|\frac{a b}{b-a} \int_{a}^{b} \frac{f(u)}{u^{2}} d u-\frac{x y}{y-x} \int_{x}^{y} \frac{f(u)}{u^{2}} d u\right| \\
& \leq \frac{M}{(a-b)(x-y)} \\
& \times\left\{b y|a-x|\left[\frac{(a-b)(x-y)}{b(x-y)-y(a-b)}\left(\ln \left|\frac{\eta(x, y)+y}{y}\right|-\ln \left|\frac{\eta(a, b)+b}{b}\right|\right)\right]\right. \\
& \left.+\frac{|a b(x-y)-x y(a-b)|}{y(a-b)-b(x-y)}\left[y(a-b) \ln \left|\frac{x}{y}\right|-b(x-y) \ln \left|\frac{a}{b}\right|\right]\right\} .
\end{aligned}
$$

Proposition 6. Let $p>1$ and $a, b, x, y \in \mathbb{R}$ with $0<a \leq x<y$ and $a<b$. Then $\eta(.,):. K \times K \rightarrow \mathbb{R}^{n}$ following inequality holds;

$$
\begin{aligned}
& \left|\frac{a b}{\eta(b, a)(p-1)}\left[\left(\frac{a b}{b+\eta(a, b)}\right)^{p-1}-a^{p-1}\right]-\frac{x y}{\eta(y, x)(p-1)}\left[\left(\frac{x y}{y+\eta(x, y)}\right)^{p-1}-x^{p-1}\right]\right| \\
& \leq \frac{M}{\eta(a, b) \eta(x, y)}\left\{b y|a-x|\left[\frac{\eta(a, b) \eta(x, y)}{b \eta(x, y)-y \eta(a, b)}\left(\ln \left|\frac{\eta(x, y)+y}{y}\right|-\ln \left|\frac{\eta(a, b)+b}{b}\right|\right)\right]\right. \\
& \left.+\frac{|a b \eta(x, y)-x y \eta(a, b)|}{y \eta(a, b)-b \eta(x, y)}\left[y \eta(a, b) \ln \left|\frac{\eta(x, y)+y}{y}\right|-b \eta(x, y) \ln \left|\frac{\eta(a, b)+b}{b}\right|\right]\right\} .
\end{aligned}
$$


Proof. If the $f(x)=x^{p}$ convex mapping defined on interval $[a, b]$ is applied to the left side of the inequality in Theorem 3 , the inequality

$$
\begin{aligned}
& \left|\frac{a b}{\eta(b, a)} \int_{a}^{\frac{a b}{b+\eta(a, b)}} \frac{f(u)}{u^{2}} d u-\frac{x y}{\eta(y, x)} \int_{x}^{\frac{x y}{y+\eta(x, y)}} \frac{f(u)}{u^{2}} d u\right| \\
& =\left|\frac{a b}{\eta(b, a)} \int_{a}^{\frac{a b}{b+\eta(a, b)}} \frac{u^{p}}{u^{2}} d u-\frac{x y}{\eta(y, x)} \int_{x}^{\frac{x y}{y+\eta(x, y)}} \frac{u^{p}}{u^{2}} d u\right| \\
& =\left|\frac{a b}{\eta(b, a)(p-1)}\left[\left(\frac{a b}{b+\eta(a, b)}\right)^{p-1}-a^{p-1}\right]-\frac{x y}{\eta(y, x)(p-1)}\left[\left(\frac{x y}{y+\eta(x, y)}\right)^{p-1}-x^{p-1}\right]\right| \\
& \leq \frac{M}{\eta(a, b) \eta(x, y)}\left\{b y|a-x|\left[\frac{\eta(a, b) \eta(x, y)}{b \eta(x, y)-y \eta(a, b)}\left(\ln \left|\frac{\eta(x, y)+y}{y}\right|-\ln \left|\frac{\eta(a, b)+b}{b}\right|\right)\right]\right. \\
& \left.\frac{|a b \eta(x, y)-x y \eta(a, b)|}{y \eta(a, b)-b \eta(x, y)}\left[y \eta(a, b) \ln \left|\frac{\eta(x, y)+y}{y}\right|-b \eta(x, y) \ln \left|\frac{\eta(a, b)+b}{b}\right|\right]\right\} .
\end{aligned}
$$

Proposition 7. Let $a, b, x, y \in \mathbb{R}$ with $0<a \leq x<y$ and $a<b$. Then $\eta(.,$.$) :$ $K \times K \rightarrow \mathbb{R}^{n}$ following inequality holds;

$$
\begin{aligned}
& \left|\frac{a b}{\eta(b, a)(-2)}\left[\left(\frac{a b}{b+\eta(a, b)}\right)^{-2}-a^{-2}\right]-\frac{x y}{\eta(y, x)(-2)}\left[\left(\frac{x y}{y+\eta(x, y)}\right)^{-2}-x^{-2}\right]\right| \\
& \leq \frac{M}{\eta(a, b) \eta(x, y)}\left\{b y|a-x|\left[\frac{\eta(a, b) \eta(x, y)}{b \eta(x, y)-y \eta(a, b)}\left(\ln \left|\frac{\eta(x, y)+y}{y}\right|-\ln \left|\frac{\eta(a, b)+b}{b}\right|\right)\right]\right. \\
& \left.+\frac{|a b \eta(x, y)-x y \eta(a, b)|}{y \eta(a, b)-b \eta(x, y)}\left[y \eta(a, b) \ln \left|\frac{\eta(x, y)+y}{y}\right|-b \eta(x, y) \ln \left|\frac{\eta(a, b)+b}{b}\right|\right]\right\} .
\end{aligned}
$$

Proof. If the $f(x)=\frac{1}{x}$ convex mapping defined on interval $[a, b]$ is applied to the left side of the inequality in Theorem 3 , the inequality

$$
\begin{aligned}
& \left|\frac{a b}{\eta(b, a)} \int_{a}^{\frac{a b}{b+\eta(a, b)}} \frac{f(u)}{u^{2}} d u-\frac{x y}{\eta(y, x)} \int_{x}^{\frac{x y}{y+\eta(x, y)}} \frac{f(u)}{u^{2}} d u\right| \\
& =\left|\frac{a b}{\eta(b, a)} \int_{a}^{\frac{a b}{b+\eta(a, b)}} \frac{u^{-1}}{u^{2}} d u-\frac{x y}{\eta(y, x)} \int_{x}^{\frac{x y}{y+\eta(x, y)}} \frac{u^{-1}}{u^{2}} d u\right| \\
& =\left|\frac{a b}{\eta(b, a)(-2)}\left[\left(\frac{a b}{b+\eta(a, b)}\right)^{-2}-a^{-2}\right]-\frac{x y}{\eta(y, x)(-2)}\left[\left(\frac{x y}{y+\eta(x, y)}\right)^{-2}-x^{-2}\right]\right| \\
& \leq \frac{M}{\eta(a, b) \eta(x, y)} \\
& \times\left\{b y|a-x|\left[\frac{\eta(a, b) \eta(x, y)}{b \eta(x, y)-y \eta(a, b)}\left(\ln \left|\frac{\eta(x, y)+y}{y}\right|-\ln \left|\frac{\eta(a, b)+b}{b}\right|\right)\right]\right. \\
& \left.+\frac{|a b \eta(x, y)-x y \eta(a, b)|}{y \eta(a, b)-b \eta(x, y)}\left[y \eta(a, b) \ln \left|\frac{\eta(x, y)+y}{y}\right|-b \eta(x, y) \ln \left|\frac{\eta(a, b)+b}{b}\right|\right]\right\} .
\end{aligned}
$$

\section{REFERENCES}

[1] Hadamard J. Étude sur les propriétés des fonctions entiéres et en particulier d'une fonction considérée par Riemann, J. Math. Pures Appl., 171-215, 58 (1893).

[2] Işcan, İ. Hermite-Hadamard type inequalities for harmonically convex functions, Hacettepe Journal of Mathematics and Statistics, Volume 43 (6), 935-942, (2014).

[3] Kilbas, A.A. Srivastava, H.M. and Trujillo, J., Theory and applications of fractional differential equations, Elsevier, Amsterdam (2006).

[4] Roberts, A.W. and Varberg, D.E. Convex Functions. Academic Press, 300pp, New York (1973).

[5] Dragomir, S.S. Cho, Y.J. and Kim, S.S., Inequalities of Hadamard's type for Lipschitzian mappings and their applications, Journal of Mathematical Analysis and Applications, vol. 245, no. 2, pp. 489-501, (2000).

[6] Hwang, S.R. Hsu, K.C. and Tseng, K.L., Hadamard-type inequalities for Lipschitzian functions in one and two variables with applications, Journal of Mathematical Analysis and Applications, vol. 405, no. 2, 546-554, (2013).

[7] İşcan, İ. New general integral inequalities for Lipschitzianfunctions via Hadamard fractional integrals, International Journal of Analysis, volume 2014, Article ID 353924, 8 pages, (2014). 
[8] İşcan, İ., Hadamard-type and Bullen-type inequalitiesfor Lipschitzian functions via fractional integrals, Mathematical Sciences and Applications E-Notes, 4 (1), 77-87, (2016).

[9] Hadamard J. " Étude sur les propriétés des fonctions entiéres et en particulier d'une fonction considérée par Riemann" , J. Math. Pures Appl., 58 , 171-215, (1893).

[10] Hanson, M.A., On sufficiency of the Kuhn-Tucker conditions, J. Math. Anal. Appl. 80, 545$550,(1981)$.

[11] İşcan, İ., Kadakal, M.,Aydın A., 'Some New integral inequalities for Lipschitzian functions' IJOCTA vol.8, no. 2, pp. 259-265, (2018).

[12] Kilinc, S., Akkurt, A., and Yıldırım, H., Integral Inequalities for Hypergeometric Operators via Fractional Integral, Journal of Inequalities \& Special Functions 8 (5), (2017).

[13] Noor, M.A., Awan, M.U. and Noor, K.I., On some inequalities for relative semi-convex functions, Journal of Inequalities and Applications, 2013.

[14] Noor, M.A., Noor, K.I. and Awan, M.U., Hermite-Hadamard inequalities for relative semiconvex functions and applications, Filomat, in press.

[15] Mohan, S.R., Neogy, S.K., On Invex Sets and Preinvex Functions, Journal of Mathematical Analysis and Applications, 1995.

[16] Noor, M.A., Awan, M.U.,Some Integral inequalities for two senses of convexities via fractional integrals, TJMM 5(2013), no.2, 129-136.

[Department of Mathematics, Faculty of Science and Arts, University of Kahramanmaraş Sütçü İmam, 46000, Kahramanmaraş, Turkey

Email address: sedakilincmath@gmail.com

[Department of Mathematics, Faculty of Science and Arts, University of Kahramanmaraş Sütçü İmam, 46000, Kahramanmaraş, Turkey

Email address: abdullahmat@gmail.com

[Department of Mathematics, Faculty of Science and Arts, University of Kahramanmaraş SütÇü İmam, 46000, Kahramanmaraş, Turkey

Email address: hyildir@ksu.edu.tr 